\title{
LAS COMPETENCIAS Y LA EVALUACIÓN DEL DESEMPEÑO DEL RECURSO HUMANO EN EL SECTOR TURÍSTICO. EL CASO DE AMBATO-ECUADOR
}

\author{
COMPETENCES AND EVALUATION OF HUMAN RESOURCE \\ PERFORMANCE IN THE TOURISM SECTOR. THE CASE OF \\ AMBATO-ECUADOR
}

Recebimento: 8/9/2016- Aceite: 11/11/2016- Publicação: 29/11/2016

Processo de Avaliação: Double Blind Review

Mary Elizabeth Cruz Lascano ${ }^{1}$

Dra. en Contabilidad y Auditoría

Dra. en Economía de la Empresa y Finanzas

Máster en Organización de Empresa

Universidad Técnica de Ambato

me.cruz@uta.edu.br

Juan Pablo Martínez Mesías

Economista.

Mg. en Administración de empresas

Universidad Técnica de Ambato

jp.martinez@uta.edu.br

Joselito Ricardo Naranjo Santamaría

Dr. Contabilidad y Auditoría.

Mg. en Costos y Gestión Financiera

Universidad Técnica de Ambato

Jr.naranjo@uta.edu.br

\footnotetext{
${ }^{1}$ Autor para correspondencia: Universidad Técnica de Ambato: Av. de los Chasquis y Río Cutuchi. Ciudadela Universitaria. Ambato-Ecuador.alexandratvalle@uta.edu.ec
} 


\title{
RESUMEN
}

La evaluación del personal es una herramienta y no un fin en sí mismo. Es un medio para obtener datos e información que puedan registrarse, procesarse y canalizarse para mejorar el desempeño humano en las organizaciones. Existen varios métodos de gestión que responden a las necesidades de la época y la estructura predominante de las organizaciones, sin embargo, el cambio es la única constante en lo que al comportamiento del ser humano se refiere, debido a esto, todo el tiempo se están analizando e investigando nuevas formas de evaluar el desempeño del personal en el ámbito empresarial, con el único fin de crear espacios de motivación que aseguren el involucramiento del personal en la consecución de las metas empresariales. En su momento, se han logrado resultados, pero aún hay camino que recorrer, el análisis de la información obtenida a través de una encuesta realizada al personal de varios establecimientos turísticos de la ciudad es la base para la determinación de la metodología de evaluación con miras a potenciar las competencias de quienes son el motor al interior de la empresa, los colaboradores.

PALABRAS CLAVE: Evaluación; Desempeño; Competencias, Eficiencia.

\begin{abstract}
Personnel evaluation is a tool and not an end in itself. It is a means to obtain data and information that can be registered, processed and channeled to improve human performance in organizations. There are several management practices that meet the needs of the time and the predominant structure of organizations, however, change is the only constant as far as human behavior is concerned, because of this, all the time are analyzing and researching new ways to evaluate staff performance in business, with the sole purpose of creating spaces of motivation to ensure the involvement of staff in achieving business goals. At the time, results have been achieved, but there is way to go, the analysis of the information obtained through a survey undertaken several staff tourist establishments in the city, will be the basis to determine the evaluation methodology in order to enhance the skills of those who are the driving force within the company, collaborators.
\end{abstract}

KEYWORDS: Evaluation; Performance; Competencies; Efficiency

ENIAC Pesquisa, Guarulhos (SP), V.5, n.2, jun.- dez. 2016 


\section{INTRODUCCIÓN}

La evaluación de las personas ha sido parte del desarrollo de la humanidad, en el área empresarial, aunque empíricamente siempre ha estado presente, se pude hacer referencia de su formalidad a partir de 1842, cuando en Estados Unidos se implantó un sistema de informes anuales para evaluar el desempeño de los empleados públicos; años más tarde, en 1880, el ejército de ese país adoptó el mismo sistema y, en 1918, empresas como la General Motors desarrollaron un sistema de evaluación para sus ejecutivos. Estas experiencias sirvieron de base para que, a partir de la segunda guerra mundial las empresas comenzaran a asumir los sistemas de evaluación del desempeño, orientados hacia la eficiencia y el aumento de la productividad. (Chiavenato, 2000)

Estos esfuerzos propiciaron que la Escuela de Administración Científica, impulse la Teoría Administrativa con el propósito de aprovechar la capacidad óptima de la máquina, a la par con el trabajo del hombre, con el fin de hacer los cálculos y lograr precisión en el rendimiento potencial en cada operación y en el funcionamiento general de la organización. En efecto, las organizaciones lograron resolver los problemas de la eficiencia y productividad en relación con las máquinas, no así con el ser humano, pues quedó pendiente el desafío ante, ¿cómo conocer y medir las potencialidades del hombre? ¿Cómo lograr que apliquen totalmente ese potencial y cuál es la fuerza fundamental que impulsa su acción?

Frente a las interrogantes anteriores de la Escuela de las Relaciones Humanas, surgen infinidad de respuestas que ocasionaron la aparición de técnicas administrativas capaces de crear condiciones para un efectivo desempeño humano en la organización y una gran cantidad de teorías sobre la motivación para el trabajo. Estas tendencias administrativas tuvieron un impacto en cuanto al papel dinamizador del hombre ante los demás recursos organizacionales; pues este papel planteaba la necesidad de implementar el desempeño humano seguido de una evaluación y orientación hacia determinados objetivos comunes organizacionales. En consecuencia, se destaca la importancia, no solo del desempeño, sino de la evaluación de éste, como un hecho cotidiano en la vida de las personas y de las organizaciones; este planteamiento desvela que la evaluación del desempeño es crucial para el mantenimiento y desarrollo de la organización.

La evaluación del desempeño de los recursos humanos, es un proceso periódico de medición que estima cualitativa y cuantitativamente el grado de cumplimiento de las actividades, objetivos y responsabilidades asignadas a las personas en sus puestos de trabajo. Busca a través de esta medición, mejorar los conocimientos, destrezas, habilidades, condiciones de trabajo, procesos y resultados.

Las empresas, objeto de este estudio pertenecen al sector turístico de la ciudad de

Ambato, la información que se obtiene de ellas permite darle pragmatismo a las percepciones sobre la realidad de un sector que no presenta un desarrollo alentador.

Su importancia radica en el papel determinante que juega el empleado, el contacto directo y

ENIAC Pesquisa, Guarulhos (SP), V.5, n.2, jun.- dez. 2016 
permanente con el cliente (turista) hace que su desempeño se refleje directamente a través de sus conocimientos, habilidades y actitudes (competencias). En el caso particular de la ciudad, el manejo formal de los recursos humanos, no es una prioridad, a pesar de la evidente necesidad. Por lo tanto, el objetivo de este trabajo es estudiar los criterios de evaluación por competencias que sirvan de marco de referencia para la evaluación integral del personal de las organizaciones a fin de mejorar los niveles de desempeño.

Para ello en primer lugar, se definen los recursos humanos y la gestión por competencias como preámbulo de comprensión de este tema; el tema siguiente es la evaluación de desempeño, los tipos y las fases de implementación y se concluye con un análisis del sector turístico de la ciudad, considerando los proyectos de incentivo turístico del gobierno provincial y los resultados de una encuesta realizada a los establecimientos del sector.

\section{ESTADO DEL ARTE \\ 1.1. La gestión de los recursos humanos}

La gestión humana, en forma progresiva y cada vez con mayor intensidad, está pasando de ser un instrumento de control administrativo, a una trascendental herramienta de competitividad sostenida que permite conocer y aprovechar las fortalezas y bondades de los trabajadores, pero más importante aún, ayuda a identificar las debilidades y temores, convirtiéndolas en oportunidades de mejora, generadoras de valor agregado y diferenciación

Tradicionalmente, el valor de la empresa se centraba en valores intangibles como las marcas y patentes y en valores tangibles, como los recursos económicos, materiales y la tecnología, pero la alta competitividad, ha evidenciado que, el adecuado aprovisionamiento de dichos recursos depende del capital humano:

El rendimiento duradero y superior, ahora requiere flexibilidad, innovación y rapidez; y la ventaja competitiva radica, en la actualidad, principalmente en los recursos internos y las capacidades de los individuos en las organizaciones”.... "uno de los factores principales, como son los recursos humanos, cobra una posición líder que posee gran influencia en otros intangibles como la clientela, la investigación, el desarrollo y la publicidad. (Becker, et al 2001: 26)

La importancia del factor humano en el desarrollo empresarial internacional, como afirma Ducci (1997: 16) “constituye una manera de recuperar la humanización del trabajo, es decir, centrar nuevamente el proceso de crecimiento económico y desarrollo social en el ser humano, como agente y beneficiario del cambio". 
Se debe recordar que los mercados de trabajo están formados por un conjunto de personas que ofrecen o demandan trabajo, que requieren acciones y reacciones frente a una serie de decisiones que afectan su interés particular como agentes económicos. Esta situación provoca, según Villasmil (2006: 14),

Que tanto empleados como empleadores busquen regular sus actuaciones a fin de conseguir condiciones favorables para las dos partes. En este sentido, Gamero (2005: 226)

Asevera que los individuos valoran de sus empleos aspectos como, el atractivo de la tarea que realizan, las condiciones ambientales en que se desarrolla, las condiciones interpersonales, la manera en que se concilia el trabajo con las actividades familiares. Esto no quiere decir que la remuneración o el factor económico pierde importancia, solo apunta a que se deben considerar otros aspectos que rodean a la actividad laboral y que pueden ser decisivos a la hora de definir la satisfacción laboral.

\section{LAS GESTIÓN DE RECURSOS HUMANOS POR COMPETENCIAS 2.1. Competencias}

De acuerdo con la Real Academia de la Lengua, competencia significa: "Pericia, aptitud, idoneidad para hacer algo o intervenir en un asunto determinado”.

El significado de competencias no es el mismo para todos, varía en función de la óptica desde la que se mire; el empresario, el colaborador, el ciudadano común, el funcionario público, el estudiante, el docente, la autoridad, etc., cada uno con objetivos propios busca alcanzar las competencias que le permitan cumplirlos, no más, de ahí que, a primera vista, muchos podrían pensar que se le ha dado mucha importancia a un tema con pocos resultados, eso se debe a la concepción que se tiene sobre el mismo, se habla de ello, pero se conoce muy poco, como manifiesta Navío (2005:216) :

\footnotetext{
La revisión conceptual nos pone de manifiesto dos cosas, por una parte la variedad de elementos que se consideran en las definiciones, por otra, la confusión terminológica que se percibe por la variedad de significados atribuidos a la competencia, especialmente en el contexto profesional y ocupacional.
}

En el análisis de la conceptualización de la competencia se hace referencia a Spenser y Spenser, quienes apuntan que la competencia es una parte duradera y profunda de la personalidad que puede predecir la conducta en una variedad de situaciones y tareas del puesto. Además la competencia permite saber quién hace algo bien o mal, medido a través de un criterio específico o estándar. Las competencias indican "formas de comportarse o pensar, que se generalizan a través de situaciones y perduran durante un período razonable de tiempo" (Spenser y Spenser, 1993:9; Agut y Grau, 2001:5). 
Si se considera que la contribución que pueden hacer las personas para alcanzar los diferentes objetivos de la empresa, la influencia que ejercen en la estructura de la empresa hasta constituirse en la clave del a diferenciación y competitividad, entonces se puede entender por qué es importante conocer todo lo que abarca el concepto de competencias. La organización del futuro se creará en torno a las personas. Si se utiliza a las personas como elementos para crear las organizaciones, éstas serán formadas en torno a lo que aportan, en otras palabras sus “competencias” (Fernández y Fajardo, 2005:18).

Adicional a ello, afirman Sánchez y Aguilera (2011:3) las relaciones y nexos que se establecen en el proceso de configuración de las competencias son cada vez más complejos y estables en la medida en que estas se aproximan al estándar establecido para ellas o sea al modo en que están configuradas, que tienen su expresión en el desempeño.

Un aspecto que se debe considerar al momento de enfocar las competencias es la relación existente entre la teoría y la práctica (Agut y Grau, 2002; BeyKan, et al, 2007). Malpica (1996), afirma que esta es más positiva cuando los conocimientos teóricos se desarrollan en la práctica identificando situaciones reales.

Desde la perspectiva de las competencias laborales se reconoce que las cualidades de las personas para desempeñarse productivamente en una situación laboral, no sólo dependen de las situaciones de aprendizaje escolar formal, sino también del aprendizaje derivado de la experiencia en situaciones concretas de trabajo. Por lo mismo, se reconoce que no bastan los certificados, títulos y diplomas para calificar a una persona como competente laboral o profesionalmente. (Huerta y Otros, 2008:2).

Una vez identificada y asumida, el desarrollo de una competencia, de acuerdo con Alles (2010: 87 y 89), se verifica luego de la puesta en práctica, es decir, con la experiencia; para que esto suceda, las personas deben tener la capacidad de analizar los éxitos y los fracasos, agregando la experiencia para acrecentar las competencias, si una persona puede conocer lo que se espera de ella en su posición y se le hace saber si su comportamiento es acorde con lo esperado, se facilita el proceso natural de desarrollo de competencias 


\subsection{La gestión por competencias}

Pereda, Berrocal y López (2002: 53) afirman que la Gestión de Recursos Humanos por Competencias surge como consecuencia de la necesidad de mantener y mejorar la competitividad de las empresas, en un entorno cada vez más agresivo y rápidamente cambiante. Cuanto más valor el trabajador tenga para la organización, más valorado estará en el mercado de trabajo (Santos, et al, 2012:357). Dicha empleabilidad aumenta en la medida que el trabajador adquiere nuevas competencias o desarrolla las que ya posee poniéndolas al servicio de la empresa (Massó, 2012:215).

Cuesta (2011: 309), concluye diciendo que la gestión de personal por competencias es una concepción actual de trascendencia para el imprescindible incremento de la productividad del trabajo, cuando se logra que el desempeño individual esté alineado con el desempeño organizacional estratégico.

Es importante resaltar que, como todo proceso en el que se requiere tomar decisiones estratégicas, la involucración del nivel directivo antes y durante la implementación del sistema es vital para obtener los resultados esperados.

Según Alles (2005:33), para trabajar con un esquema de competencias es necesario:

- Definir la misión y visión de la empresa (si aún no la tiene).

- Definición de competencias de la máxima dirección de la compañía.

- Prueba de competencias en un grupo de ejecutivos de la organización.

- Validación de las competencias.

- Diseño de procesos de recursos humanos por competencias.

Figura 1. Sistema de Gestión por Competencias
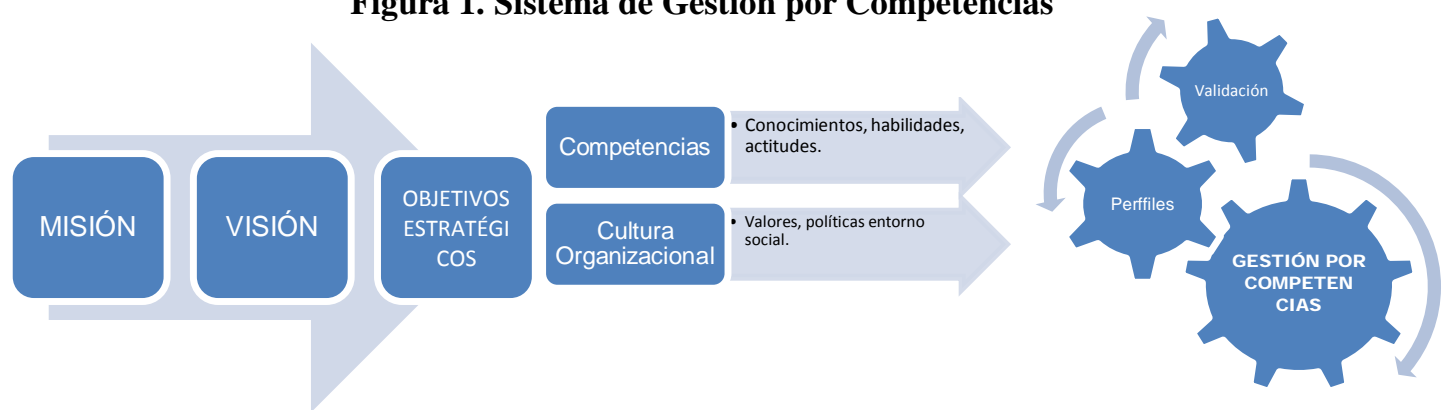

Fuente: Alles (2005:33)

Elaboración propia

Mertens (1998: 47), plantea los componentes básicos de un sistema de competencias: 
- Crear un ambiente en el que pueda emerger la competencia laboral.

- Describir las normas de competencia por subprocesos y/o funciones.

- Desarrollar currículo.

- Formar en base a competencias.

- Diseñar un plan de evaluación del personal.

Estos elementos que facilitan la gestión por competencias, deben desembocar en todo el proceso de gestión de talento humano clásico de selección, compensación, desarrollo, mantenimiento y evaluación, con énfasis en éste último, pues a través de su aplicación se define si el sistema aporta a la consecución de los objetivos organizacionales.

\section{LA EVALUACIÓN DEL DESEMPEÑO}

La evaluación del desempeño ha sido y seguirá siendo un tema que provoca muy diversos criterios debido a las diferencias de conceptos y experiencias tanto de evaluadores, evaluados, expertos en el tema, empresarios y observadores externos.

Lo único en lo que la mayoría está de acuerdo es en la necesidad de realizarla. Las discrepancias las encontramos en los métodos de aplicación, en las formas de determinar los resultados e interpretación de los mismos y más aún, en los efectos que estos resultados provocan, ya sea por falta de preparación, distorsión en la comprensión, o falta de compromiso de la dirección. Cuando no se realiza adecuadamente, los resultados no cubren las expectativas iniciales.

Siendo este un aspecto que puede generar motivación y desarrollo en una organización y, a la vez, todo lo contrario, desmotivación y estancamiento en las relaciones internas, es necesario comprender con claridad qué significa realmente. La evaluación del desempeño no puede restringirse a un simple juicio superficial y unilateral del jefe respecto del comportamiento funcional del subordinado; es necesario descender más profundamente, localizar las causas y establecer perspectivas de común acuerdo con el evaluado (Chiavenato, 2000: 104).

La evaluación del desempeño es un proceso sistemático, continuo, orgánico y en cascada, de expresión de juicios acerca del personal de una empresa, en relación con su trabajo habitual, que pretende sustituir a los juicios ocasionales y formulados de acuerdo con los más variados criterios. La evaluación tiene una óptica histórica (hacia atrás) y prospectiva (hacia adelante) y pretende integrar en mayor grado los objetivos organizacionales con los individuales. (Puchol, 2003:89) 
Se la cataloga también una herramienta de competitividad, al definirla como:

\author{
Un proceso para evaluar formalmente la conducta laboral y proporcionar una \\ retroalimentación en la cual pueden hacerse los ajustes en la misma, contribuye \\ con la administración de la empresa porque es un medio para que una \\ organización mantenga su productividad y optimice sus recursos \\ humanos.(Grados, Beutelspacher y Castro, 2002: 15).
}

Sin embargo, no existe una receta única, cada empresa deberá determinar el método de evaluación que se ajuste a sus necesidades. Para Bohlander y Snell (2008: 376,377), la elección del método se debe basar sobre todo en el propósito de la evaluación. (...). Aunque los investigadores y gerentes de recursos humanos, por lo general, creen que los métodos que son más sofisticados y que ocupan más tiempo ofrecen información más útil, éste no siempre es el caso. Una manera de evaluar si el sistema de evaluación de una organización es efectivo es realizar una auditoría del proceso cada año, o por lo menos periódicamente.

Hay que considerar que tanto los empleados como las organizaciones tienen objetivos específicos para el proceso de la evaluación del desempeño (Rodríguez, 2007: 387), por ello para que la evaluación no se torne inalcanzable, señala González (2006: 271), se debe asegurar con la gerencia la consecución de los recursos y medios necesarios para alcanzar los objetivos con eficacia.

El proceso de la evaluación del desempeño tiene cuatro fases:

1. Fase de definición de objetivos

2. $\quad$ Fase de diseño

3. $\quad$ Fase de implantación

4. $\quad$ Fase de control y evaluación

\title{
3.1. Evaluación por competencias
}

El núcleo de la evaluación por competencias está en la definición de las competencias y su interpretación estandarizada a través de un diccionario de competencias; y, la determinación de los perfiles de cargo que incluyan los requisitos de conocimientos, habilidades y actitudes (competencias), que debe cubrir cada uno. 
La objetividad de su aplicación está condicionada por la experticia de los evaluadores y el establecimiento de estándares y evidencias contra las cuáles se debe evaluar el cumplimiento de los requisitos especificados.

\section{METODOLOGÍA}

El estudio tiene un enfoque cuali-cuantitativo y es de tipo descriptivo. La modalidad básica de investigación es la de campo, se recopilan datos directamente del universo de estudio, constituyéndose en una fuente primaria, a través de una encuesta aplicada en el período de julio a septiembre de 2012.

La encuesta tiene dos partes, la primera que recoge datos de tipo general, y la segunda que tiene su base en el conjunto de normas de competencias para el sector turístico homologadas para el Ecuador. Se dirigió los hoteles y restaurantes de la provincia. Del listado de empresas solicitado al S.R.I. ${ }^{2}$, se escogieron al azar 50 empresas, para encuestar a sus empleados, resultando de ellas un número previo de 350 encuestas válidas

\section{RESULTADOS Y DISCUSIÓN}

\subsection{El Turismo Ambato.}

Por su ubicación, Tungurahua se distingue por ser vértice de movilidad. Esta particularidad ha dado a la provincia, especialmente a su capital Ambato, un rol históricamente sólido como uno de los principales nodos articuladores y distribuidores del flujo comercial dentro del país. En los últimos años la centralidad espacial, -Ambato está pubicada en el centro del país- ha sido reforzada por una red vial que atraviesa su territorio y que en términos comparativos es de las más densas y de mejor calidad.

Esta característica comercial, beneficiosa para el movimiento económico de la provincia, hace que la actividad turística, en cambio, sea secundaria, tanto en generación de producto bruto como en absorción de fuerza laboral.

La ciudad de Ambato, capital de la provincia del Tungurahua se encuentra ubicada a 2.557 metros de altitud, con una superficie de 1.008,8 Km2.

En la tabla 1 se recoge lo relativo a los recursos humanos en el documento Estrategia de Turismo para la Provincia de Tungurahua realizado por el Gobierno Provincial:

${ }^{2}$ Servicio de Rentas Internas. 
TABLA 1. Los Recursos Humanos en el Sector Turístico de Ambato

RESTRICCIONES

- Conocimiento inadecuado del mercado turístico relevante, que no permite la segmentación de productos para ofrecerlos al turista.

- Deficiente cultura de servicio al turista y calidad de servicios. FORTALEZAS

- Presencia de Universidades activas en el campo de formación de recurso humano para la actividad turística.

- Presencia de una planta turística diversificada especialmente en hotelería y restaurantes LINEA ESTRATÉGICA: Capacitación y formación de habilidades.

\section{ACCIONES:}

- Establecer una agenda cantonal de capacidades y habilidades requeridas para el desarrollo turístico cantonal.

- Desarrollar procesos de formación de capacidades y habilidades para la actividad turística.

- Desarrollar iniciativas de concienciación y promoción de la actividad turística.

Fuente: Elaboración propia a partir de Estrategia de turismo de la provincia de Tungurahua. Gobierno Provincial y Otros.

http://www.tungurahua.gob.ec/turismo/index.php?option=com content\&view=article\&id=174\&Itemid=144

En lo referente a las variables socio-demográficas, la información resumida se presenta en la tabla 2:

Tabla 2. Resumen de variables socio-demográficas

\begin{tabular}{|c|c|c|c|}
\hline VARIABLE & ITEMS & NÚMERO & PORCENTAJE \\
\hline \multirow[t]{3}{*}{ Sexo } & Hombre & 157,0 & 44,9 \\
\hline & Mujer & 193,0 & 55,1 \\
\hline & Total & 350,0 & 100,0 \\
\hline \multirow{4}{*}{ Edad } & 18 а 30 & 131,0 & 66.8 \\
\hline & 31 a 50 & 189,0 & 31.5 \\
\hline & 51 a 65 & 30,0 & 1.7 \\
\hline & Total & 350,0 & 100,0 \\
\hline \multirow{5}{*}{ Estado Civil } & Soltero & 217,0 & 62,0 \\
\hline & Casado & 107,0 & 30,6 \\
\hline & Divorciado & 23,0 & 6,6 \\
\hline & Unión Libre & 3,0 & 0,9 \\
\hline & Total & 350,0 & 100,0 \\
\hline \multirow{5}{*}{ Formación } & $\begin{array}{l}\text { Escuela } \\
\text { Primaria }\end{array}$ & 45 & 12.9 \\
\hline & Ciclo Básico & 44 & 12,6 \\
\hline & Bachillerato & 134 & 38,3 \\
\hline & Superior & 127 & 34,3 \\
\hline & Total & 350,0 & 100,0 \\
\hline \multirow{7}{*}{$\begin{array}{l}\text { Área de } \\
\text { Formación }\end{array}$} & Turismo & 59 & 16,9 \\
\hline & Administración & 80 & 22,9 \\
\hline & Financiera & 28 & 8,0 \\
\hline & Sistemas & 19 & 5,4 \\
\hline & Otra & 84 & 24,0 \\
\hline & N/A & 80 & 22,9 \\
\hline & Total & 350,0 & 100,0 \\
\hline
\end{tabular}

Fuente: Elaboración propia a partir de los datos de la encuesta. 
Se puede observar que, en esta muestra del sector, los hombres tienen una menor incidencia en los cargos pues representan un $44,9 \%$ de la población; la edad predominante, con un 66,8\% , es la comprendida entre 18 y 30 años, un segmento joven; y el estado civil de la mayoría de los encuestados es el de soltero con un 62\%. En cuanto al nivel de educación de los encuestados está compartido entre el bachillerato con un 38,3\% y la educación superior con un 34,3\%; sin embargo, es importante anotar que el $25 \%$ de los encuestados solo ha llegado a cursar la primaria y el ciclo básico. El área de formación, en cambio, es bastante dispersa, el 32\% corresponde a otras profesiones no relacionadas.

Los cargos, definidos conforme a las normas mencionadas en la tabla 6, se distribuyen entre los encuestados de acuerdo a la tabla 3 :

Tabla 3. Frecuencia de la variable cargos.

\begin{tabular}{|l|r|r|}
\hline \multicolumn{1}{|c|}{ VARIABLE: CARGOS } & FRECUENCIA & PORCENTAJE \\
\hline Administrador de Restaurante & 95 & 27,1 \\
\hline $\begin{array}{l}\text { Administrador de Empresas de } \\
\text { Alojamiento }\end{array}$ & 12 & 3,4 \\
\hline Recepcionista & 19 & 5,4 \\
\hline Cajero & 55 & 15,7 \\
\hline Chef de cocina & 6 & 1,7 \\
\hline Cocinero & 57 & 16,3 \\
\hline Mesero & 53 & 14,9 \\
\hline Posillero & 4 & 1,4 \\
\hline Vendedor & 7 & 2,0 \\
\hline Botones & 1 &, 3 \\
\hline Ama de Llaves & 1 &, 3 \\
\hline Ayudante de mesero & 2 &, 6 \\
\hline Agente de Ventas & 14 & 4,0 \\
\hline Camarera de pisos & 10 & 2,9 \\
\hline Gerente de Operadora & 7 & 2,0 \\
\hline Encargado de Mantenimiento & 4 &, 9 \\
\hline Otro & 3 & 1,1 \\
\hline Total & $\mathbf{3 5 0}$ & \\
\hline Fun & &
\end{tabular}

Fuente: Elaboración propia a partir de los datos de la encuesta.

En esta muestra, los cargos con mayor incidencia son: el de administrador de restaurante con un $27,1 \%$, el de cocinero polivalente con un $16,3 \%$ y el de mesero con un $14,9 \%$, seguidos por el cargo de recepcionista con un 5,4\%. 
Cabe hacer la aclaración, los Administradores de restaurant son, en su mayoría, profesionales de otras ramas que decidieron incursionar en esta rama por la liquidez y movimiento de capital que este tipo de negocio genera, además por los márgenes de rentabilidad que se manejan, pero la informalidad en el área de personal hizo que las encuestas se las pudiera aplicar a ellos, restringiendo en muchos casos, el acceso a sus empleados, por ello el número importante de este cargo.

En lo referente a la variable sobre la evaluación del desempeño, se realizaron tres preguntas, cuyos resultados aparecen recogidos en la tabla 4:

Tabla 4. Resultados encuesta sobre evaluación de desempeño.

\begin{tabular}{|l|c|c|c|c|c|c|c|c|}
\hline \multicolumn{1}{|c|}{ PREGUNTA } & \multicolumn{2}{c|}{ SI } & \multicolumn{2}{c|}{ NO } & \multicolumn{2}{c|}{ N/A } & \multicolumn{2}{c|}{ TOTAL } \\
\cline { 2 - 9 } & $\mathrm{N}^{\circ}$ & $\mathrm{F}$ & $\mathrm{N}^{\circ}$ & $\mathrm{F}$ & $\mathrm{N}^{\circ}$ & $\mathrm{F}$ & $\mathrm{N}^{\circ}$ & $\mathrm{F}$ \\
\hline $\begin{array}{l}\text { Ha sido evaluado sobre su } \\
\text { desempeño en el trabajo? }\end{array}$ & 143 & 40,9 & 207 & 59,1 & - & - & 350 & 100,0 \\
\hline $\begin{array}{l}\text { Conoce el resultado de esas } \\
\text { evaluaciones? }\end{array}$ & 81 & 23,1 & 64 & 18,3 & 205 & 58,6 & 350 & 100,0 \\
\hline $\begin{array}{l}\text { Cree Ud. que las evaluaciones } \\
\text { han generado mejoras para su } \\
\text { cargo? }\end{array}$ & 115 & 32,9 & 10 & 2,6 & 225 & 64,5 & 350 & 100,0 \\
\hline
\end{tabular}

Del total de la población, el 40,9 \% ha sido evaluado de alguna manera. De este grupo, solo el 23,1\% ha conocido los resultados, pero aún así, el $80 \%$ de los que respondieron haber sido evaluados opinan que las evaluaciones les han generado mejoras en los cargos, aún cuando no todos conocieron los resultados.

Es también importante resaltar que más del 50\% de los empleados encuestados, no han sido evaluados, este dato está influenciado por el gran número de administradorespropietarios de los restaurantes que, por obvias razones, no son evaluados, pues están arriba en la jerarquía de sus empresas.

Para cada cargo mencionado en la tabla 8 se preparó una encuesta con preguntas basadas en las competencias que se presentan en la Norma INEN correspondiente, a modo de ejemplo y por tratarse de un cargo de marcado contacto con el cliente, se han escogido los cargos de administrador de restaurante, mesero y recepcionista, para presentar los resultados en las tablas 5, 6 y 7 respectivamente.

Las competencias de cada cargo están divididas en tres categorías: Conocimientos, habilidades y actitudes, y éstas en conjunto, están en un rango de 15 a 20 ítems, por ello, para facilitar la comprensión y no abundar en información, a modo de ejemplo, solo se escogieron de los resultados, tres ítems, uno por cada categoría. Estos ítems fueron escogidos al azar. 
Tabla 5. Resultados sobre las competencias para el cargo de administrador de restaurante.

\begin{tabular}{|l|c|c|c|c|c|c|c|c|c|c|c|c|c|}
\hline \multirow{2}{*}{ COMPETENCIA } & \multicolumn{2}{|c|}{ Nada } & \multicolumn{2}{c|}{$\begin{array}{c}\text { Casi } \\
\text { nada }\end{array}$} & \multicolumn{2}{c|}{ Poco } & \multicolumn{2}{c|}{ Bastante } & \multicolumn{2}{c|}{ Mucho } & \multicolumn{2}{c|}{ Total } \\
\cline { 2 - 21 } & $\mathbf{N}^{\circ}$ & $\mathbf{F}$ & $\mathbf{N}^{\circ}$ & $\mathbf{F}$ & $\mathbf{N}^{\circ}$ & $\mathbf{F}$ & $\mathbf{N}^{\circ}$ & $\mathbf{F}$ & $\mathbf{N}^{\circ}$ & $\mathbf{F}$ & $\mathbf{N}^{\circ}$ & $\mathbf{F}$ \\
\hline $\begin{array}{l}\text { Procesos básicos de liderazgo y } \\
\text { gestión de personal. } \\
\text { (Conocimiento) }\end{array}$ & 2 & 2,1 & 7 & 7,3 & 33 & 34,7 & 34 & 35,7 & 19 & 35 & 95 & 100 \\
\hline $\begin{array}{l}\text { Toma de decisiones en } \\
\text { situaciones críticas con los } \\
\text { clientes (habilidad) }\end{array}$ & & & 3 & 3,1 & 4 & 4,2 & 36 & 37,9 & 52 & 54,8 & 95 & 100 \\
\hline $\begin{array}{l}\text { Confiable, hace que otros se } \\
\text { sientan cómodos. (actitud). }\end{array}$ & & & 3 & 3,1 & 4 & 4,2 & 21 & 21,9 & 67 & 70,8 & 95 & 100 \\
\hline
\end{tabular}

Fuente: Elaboración propia a partir de los datos de la encuesta

Tabla 6. Resultados sobre las competencias para el cargo de Mesero

\begin{tabular}{|l|c|c|c|c|c|c|c|c|c|c|c|c|c|}
\hline \multirow{2}{*}{\multicolumn{1}{|c|}{ COMPETENCIA }} & \multicolumn{2}{|c|}{ Nada } & \multicolumn{2}{c|}{$\begin{array}{c}\text { Casi } \\
\text { nada }\end{array}$} & \multicolumn{2}{c|}{ Poco } & \multicolumn{2}{c|}{ Bastante } & \multicolumn{2}{c|}{ Mucho } & \multicolumn{2}{c|}{ Total } \\
\cline { 2 - 14 } & $\mathbf{N}^{\circ}$ & $\mathbf{F}$ & $\mathbf{N}^{\circ}$ & $\mathbf{F}$ & $\mathbf{N}^{\circ}$ & $\mathbf{F}$ & $\mathbf{N}^{\circ}$ & $\mathbf{F}$ & $\mathbf{N}^{\circ}$ & $\mathbf{F}$ & $\mathbf{N}^{\circ}$ & $\mathbf{F}$ \\
\hline $\begin{array}{l}\text { Vocabulario técnico relativo al } \\
\text { servicio de alimentos y } \\
\text { bebidas. (conocimiento) }\end{array}$ & 1 & 1,9 & 3 & 5,7 & 7 & 13,2 & 14 & 35 & 28 & 55 & 53 & 100 \\
\hline $\begin{array}{l}\text { Integración para ejecutar } \\
\text { trabajo en equipo. (habilidad) }\end{array}$ & & & & & 1 & 1,9 & 16 & 30,2 & 36 & 67,9 & 53 & 100 \\
\hline $\begin{array}{l}\text { Cordial con el cliente, } \\
\text { considerado hacia los otros. } \\
\text { (actitud). }\end{array}$ & & & & & & & 10 & 18,9 & 43 & 81,1 & 53 & 100 \\
\hline
\end{tabular}

Fuente: Elaboración propia a partir de los datos de la encuesta.

Tabla 7. Resultados sobre las competencias para el cargo de Recepcionista

\begin{tabular}{|l|c|c|c|c|c|c|c|c|c|c|c|c|}
\hline \multirow{2}{*}{ COMPETENCIA } & \multicolumn{2}{|c|}{ Nada } & \multicolumn{2}{|c|}{$\begin{array}{c}\text { Casi } \\
\text { nada }\end{array}$} & \multicolumn{2}{c|}{ Poco } & \multicolumn{2}{c|}{ Bastante } & \multicolumn{2}{c|}{ Mucho } & \multicolumn{3}{c|}{ Total } \\
\cline { 2 - 14 } & $\mathbf{N}^{\circ}$ & $\mathbf{F}$ & $\mathbf{N}^{\circ}$ & $\mathbf{F}$ & $\mathbf{N}^{\circ}$ & $\mathbf{F}$ & $\mathbf{N}^{\circ}$ & $\mathbf{F}$ & $\mathbf{N}^{\circ}$ & $\mathbf{F}$ & $\mathbf{N}^{\circ}$ & $\mathbf{F}$ \\
\hline $\begin{array}{l}\text { Procedimientos de control } \\
\text { relacionados con el acceso a las } \\
\text { habitaciones. (conocimiento) }\end{array}$ & & & & & & & 3 & 15,8 & 16 & 84,2 & 19 & 100 \\
\hline $\begin{array}{l}\text { Interpretación del lenguaje } \\
\text { corporal. (habilidad) }\end{array}$ & & & & & & & 8 & 42,1 & 11 & 57,9 & 19 & 100 \\
\hline $\begin{array}{l}\text { Cordial con el cliente, } \\
\text { considerado hacia los otros. } \\
\text { (actitud). }\end{array}$ & 3 & 15,7 & & & & & 6 & 31,6 & 10 & 56,7 & 19 & 100 \\
\hline
\end{tabular}

Fuente: Elaboración propia a partir de los datos de la encuesta.

Los resultados, en este caso, mantienen la tendencia de todos los cargos, con las lógicas variaciones dependiendo del mismo. Para las competencias relativas al conocimiento se tienen porcentajes tendentes a los valores menores; cuando se trata de 
habilidades éstas tienen mejores resultados y con respecto a las actitudes los resultados mejoran. Esto nos permite comprobar la informalidad en la educación y la experiencia que adquieren en base a la práctica.

Son evidentes los beneficios que se obtendrían al generar, difundir e implementar sistemas de gestión de recursos humanos por competencias en el sector, el trabajo está pendiente y el sector está dispuesto porque ha identificado el problema. Se necesita el empuje de instituciones de educación y formación como las Universidades y centros de estudio para llevarlo a cabo.

\section{CONCLUSIONES}

La evaluación del desempeño ha sido, es y será un tema que genera diversidad de criterios y resultados que, lógicamente, se dan en función de las experiencias generadas antes, durante y después de su aplicación. En muchos casos, se adopta esta herramienta de gestión por razones que no guardan relación con el verdadero sentido de su utilización, puede ser por cumplir un requisito, simplemente porque todas las empresas lo hacen o por decisión directiva, sin involucramiento. Cuando esto ocurre las situaciones que se suscitan y los resultados que se obtienen muy pocas veces cubren las expectativas, muy al contrario pueden incluso llegar a complicar aquello que se pretendía solucionar.

Existen diversos métodos de evaluación, que dependiendo del tipo de empresa y la metodología de aplicación, pueden funcionar adecuadamente, esto quiere decir que el responsable del área de recursos humanos debe analizar previamente el ambiente laboral, los errores que se podrían producir, el tipo de dirección, la disposición de inversión y los conocimientos de los mandos altos y medios, para elegir y aplicar un método que admita tomar decisiones que permitan conseguir las metas iniciales. Se pueden combinar varios métodos o crear uno que se ajuste a las necesidades propias de la organización, todo depende de las competencias del equipo responsable.

Actualmente, existen nuevas tendencias de evaluación, que buscan aprovechar y mejorar continuamente las habilidades, conocimientos y destrezas de los colaboradores, a través de la fijación de objetivos y la evaluación permanente de su cumplimiento basado en el desempeño de los empleados. Esto hace que sea catalogada como una herramienta de competitividad, si es aprovechada.

La evaluación del desempeño por competencias es la elección actual de las empresas, ya sea porque se encuentran implantando sistemas de calidad, porque cuentan con normas de competencias para los cargos de su sector o porque otras empresas que han implementado este sistema están obteniendo buenos resultados y esto constituye una motivación. Además, se ha generado y se continúa generando mucha información sobre el 
tema, que aporta a que día a día se vayan sumando más organizaciones a la utilización de esta herramienta de gestión y evaluación.

La situación del sector turístico de la provincia de Tungurahua está empezando a ser esperanzadora puesto que se ha identificado que la cualificación y la formación de los recursos humanos es una debilidad importante y el Estado ha impulsado y desarrollado un proyecto para normalizar las competencias de los cargos del sector; sin embargo, aunque los elementos para mejorar esta situación se han dado, la concreción de las actividades a través de un sistema de gestión por competencias aún no ha llegado. Aquí tenemos, entonces, una oportunidad de fusionar estas herramientas y mejorar sustancialmente el desempeño de los trabajadores del sector.

\section{BIBLIOGRAFÍA}

Agut N., s. Y Grau G., R. (2001). “Una aproximación psicosocial al estudio de las competencias”, Proyecto Social: Revista de relaciones laborales, n 9, págs. 13-24

Alles, Martha (2005). Gestión por Competencias. El Diccionario, Editorial Granica, Buenos Aires.

Alles, Martha (2010). Desarrollo del Talento Humano. Basado en competencias, Editorial Granica, 2da. Edición, Buenos Aires.

Becker, B.; Huselid, A. y Ulrich, D. (2001). Cuadro de Mando de Recursos Humanos en la Empresa, Ed. Gestión 2000, Barcelona.

Beykan, Cizel, Nilgün Anafarta, Fulya, Sarvan (2007), An analysis of managerial competency needs in the tourism sector: the case of Turke, vol. 62, $n^{\circ} 2$, pags. 14-22.

Bohlander, G. y Snell, S. (2008): Administración de Recursos Humanos, Cengage Learning Editores, México. 14 a edición.

Chiavenato, I. (2000). Administración de recursos humanos, McGraw Hill, Bogotá, 5a edición.

Cuesta, A. (2011). "Metodología de gestión por competencias asumiendo la norma cubana sobre gestión de capital humano”, RBGN-Revista Brasileña de Gestión de Negocios, vol. 13, n 40, págs. 300-311. 
Ducci, María Angélica. (1997). El Enfoque de Competencia Laboral en la Perspectiva Internacional. Formación basada en competencia Laboral. CINTERFOR/OIT. Montevideo.

Fernandez, Guadalupe y Fajardo, Plácido. (2005). Las Competencias: Clave para una gestión integrada de los recursos humanos. Ediciones Deusto. Barcelona. España.

Gamero B., Carlos. (2005): “Análisis Microeconómico de la Satisfacción Laboral”, CES Colección Estudios, $n, \mathrm{n}^{\circ}$ 66, págs. 226-229.

Grados, J.; Beutelspacher, O. y Castro, M. (2002): Calificación de Méritos. Evaluación de Competencias Laborales, Rodefi Impresores S.A., México.

Huerta, Jesús y Otros (2008). "Desarrollo curricular por competencias profesionales integrales”. Revista Educar - colaboración.uv.mx. 01/09/2008, http://educacion.jalisco.gob.mx/consulta/educar/13/13Huerta

Malpica, María del Carmen, (1996). "El punto de vista pedagógico”, en Arguelles, A., op. cit., págs. 123-140.

Massó, Matilde; Lozares, Carlos. (2012). “Un análisis de la gestión de las competencias en el trabajo, el caso de los mandos intermedios de una factoría de carrocería y montaje de automóviles”, Cuadernos de Relaciones Laborales, vol. 30, n 1, págs. 211-233.

Mertens, Leonard. (1998). “La Gestión por Competencia Laboral en la Empresa y la Formación Profesional”. Organización de Estados Iberoamericanos para la Educación, la Ciencia y la Cultura (OEI). Madrid.

Navío Gámez, Antonio (2005). "Propuestas conceptuales en torno a la competencia profesional”. Revista de Educación, $n^{\circ}$ 337, págs. 213-234, Universidad Autónoma de Barcelona. España.

Pereda, S.; Berrocal, F. y López, M. (2002): “Gestión de Recursos Humanos por Competencias y Gestión del Conocimiento”. Revista Dirección y Organización DYO, n 28, págs. 28-54.

Puchol, L. (2007), Dirección y gestión de recursos humanos, Ediciones Días de Santos, $4^{\mathrm{a}}$ edición, España.

Spenser, L.M. y Spenser, S.M. (1993): Competence at work. Models for superior performance, John Wiley \& Sons Inc., New York.

ENIAC Pesquisa, Guarulhos (SP), V.5, n.2, jun.- dez. 2016 
Sánchez P. y Aguilera E. (2011). "Hacia una fundamentación de la configuración de competencias profesionales”. Revista Universidad y Sociedad, vol. $3, \mathrm{n}^{\circ} 1$. Universidad de Cienfuegos Carlos Rafael Rodríguez. Enero- Abril. Cuba.

Santos, F.; Guillén, C.; y Montalbán, M. (2012). "Contrato de trabajo, compromiso y satisfacción. Moderación de la empleabilidad”, RAE, Sao Paulo, vol. 52, n 3, mayo-junio, págs. 345-359.

Villasmil, M. A. (2006): "Una política de articulación para la formación de capital humano y las políticas de empleo aplicadas al sector hotelero”, Revista Visión Gerencial, Año 5, n 2, págs. 213-236 